\title{
Analysis of Fatigue Crack Initiation and Propagation in Cold Forging Tools by Local Approach of Fracture*
}

\author{
Kunio Hayakawa, Tamotsu Nakamura and Shigekazu Tanaka \\ Department of Mechanical Engineering, Shizuoka University, Hamamatsu 432-8561, Japan
}

The local approach of fracture based on continuum damage mechanics (CDM) combined with the finite element method (FEM) was applied to establish a more precise method of estimation of service life of cold forging tools by simulating the initiation and growth of a fatigue crack. After a brief explanation on the local approach of fracture based on CDM combined with FEM, modeling of elastic-plastic-damage behavior of tool steel was conducted in the framework of thermodynamics. In the modeling, the salient unilateral property of the tool material is considered by introducing a modified stress tensor. The proposed constitutive equations can be found to describe the experimental behaviors under uniaxial tension and compression properly. Then, the initiation and propagation of a fatigue crack in the cold forward extrusion die was analyzed by the local approach of fracture. The initiation of the fatigue crack in the vicinity of the die radius was found to occur when the number of extrusion increased. Then, the crack propagated along the direction perpendicular to the surface of the die radius. Furthermore, the proposed approach was found to estimate the actual behavior of the fatigue crack growth in good agreement with the calculated change of the rate of crack propagation to the number of extrusion. The calculated crack propagation rate also tended to decrease due to a decrease in tensile principal stress at the crack tip as the crack propagates.

(Received June 4, 2003; Accepted November 6, 2003)

Keywords: fatigue crack, forging tool, finite element method (FEM), elastic-plastic material, continuum damage mechanics, cold forward extrusion

\section{Introduction}

Cold forging is one of the most essential production methods for producing net and near-net-shape parts such as shafts, axles, bolts, gears and joints.

The considerable stress on tools and workpieces is one of the most characteristic problems in most cold forging operations. The cyclic loading of the tools during cyclic forming operations may cause fatigue cracks, which lead to a limited tool life.

In cold forging, the establishment of a method for the exact estimation of the service life of forging tools is one of the most important objectives in the reduction of the total cost of the operations due to unexpected failure of the dies during the manufacturing operations. Therefore, several experimental and numerical studies on the fracture and estimations of service life of forging tools have been carried out to date. ${ }^{1-9)}$

As far as the numerical studies on the estimation of the service life of forging tools are concerned, some studies on finite element analysis of the fatigue crack growth of forging tools have been performed. ${ }^{6-8)}$ The Paris law that relates the rate of the fatigue crack with the range of the stress intensity factor based on the fracture mechanics (FM) has been used in these analyses. As far as the numerical studies on the fatigue crack by FM and the finite element method (FEM) are concerned, the initiation and growth of the fatigue crack must be treated separately. Moreover, the preexisting macroscopic cracks must be assumed in order to calculate the range of stress intensity factor, and the use of the special quarter-node elements at the crack tip is necessary for the exact calculation of the strong stress singularity.

Hänsel et al. ${ }^{3)}$ refer to the initiation of the fatigue crack of the cold forging die in their research. They used the

\footnotetext{
*This Paper was Originally Published in Japanese in J. Jpn. Soc. Technol. Plasticity, 43-497 (2002), 546-550.
}

dissipation energy by the local stress-strain hysteresis as a threshold value of crack initiation. However, they also treated the crack growth separately from the crack initiation.

On the other hand, the continuum damage mechanics (CDM) is capable of describing the whole process of failures ranging from damage development, crack initiation, crack (or failure zone) growth to the final fracture. ${ }^{10-12)}$ Namely, if we characterize a crack by an aggregate of material elements where the damage variable attains to a critical value, we can analyze the whole process of the initiation and growth of cracks. This scheme is usually called the local approach of fracture. Because of its potential capability in engineering applications, the local approach of fracture based on CDM combined with FEM has been extensively investigated in fields of engineering.

In the present paper, the local approach of fracture based on CDM combined with FEM is applied to simulate the initiation and growth of the fatigue crack of cold forging tools, to establish a more precise method of estimation of the service life of the tools.

First, the framework of the local approach of fracture based on CDM combined with FEM is described briefly. Next, elastic-plastic-damage constitutive equations for the cold tool material that has the salient unilateral characteristic of the material are formulated based on the framework of the irreversible thermodynamic theory. Then, FE analysis of the local approach of fracture based on CDM combined with FEM of the fatigue crack initiation and growth of the die used in cold forward extrusion are performed.

\section{Local Approach of Fracture with $\mathrm{CDM}^{10-12)}$}

The local approach of fracture is a method of predicting the behavior of cracks in structures by means of continuum mechanics concepts defined in terms of the elementary volume element only. Such a material point may have 


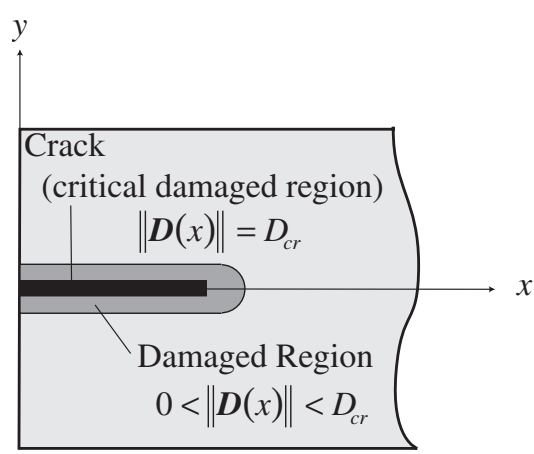

Fig. 1 Concept of local approach of fracture.

properties of elasticity, plasticity, and viscosity and may be damaged until it breaks, leading to initiation of macroscopic cracks.

Therefore, if the constitutive behavior of the damaged materials can be described by the concept of CDM, the behavior of cracks may be obtained by structure calculations, e.g. such as FEM, without any reference to concepts.

Figure 1 shows the concept of the local approach of fracture with CDM. In the procedure of CDM, the damage state at a point $x$ in the material is described by the damage variable $\boldsymbol{D}(x)$. Thus, if we characterize a crack by an aggregate of material elements where the certain norm of damage variable $\|\boldsymbol{D}(x)\|$ at the material point $x$ attains to a critical value $D_{c r}$ as shown in Fig. 1, we can analyze the process of initiation and growth of the crack.

The local approach of fracture based on CDM combined with FEM has been extensively investigated in fields of engineering such as creep fracture, ductile fracture, brittle fracture and low cyclic fatigue.

\section{Constitutive Equations of Tool Materials}

In the present study, we use cold tool steel JIS SKD11 (AISI D2) as a tool material. The behavior of the material is modeled by the elastic-plastic constitutive equations coupled with damage based on the framework of CDM with the irreversible thermodynamics theory for the constitutive equation. ${ }^{13,14,16)}$

In the present study, the strain of the tool material $\varepsilon$ can be decomposed into the elastic and the plastic parts as follows, as the deformation of the forging tools can be assumed infinitesimal.

$$
\boldsymbol{\varepsilon}=\boldsymbol{\varepsilon}^{e}+\boldsymbol{\varepsilon}^{p}
$$

\subsection{Material damage and damage variable}

The cold tool steel is commonly used after the heat treatment to obtain the adequate hardness by the precipitation of the carbides in the matrix. However, these precipitated carbides may cause stress concentration. Furthermore, the debonding of the interface between the carbides and the matrix, and the fracture of the carbides themselves also cause the microscopic material damage.

The initiation and growth of the material damage depends the direction of the applied stress. Moreover, the effects of the damage are diminished or reduced under compression

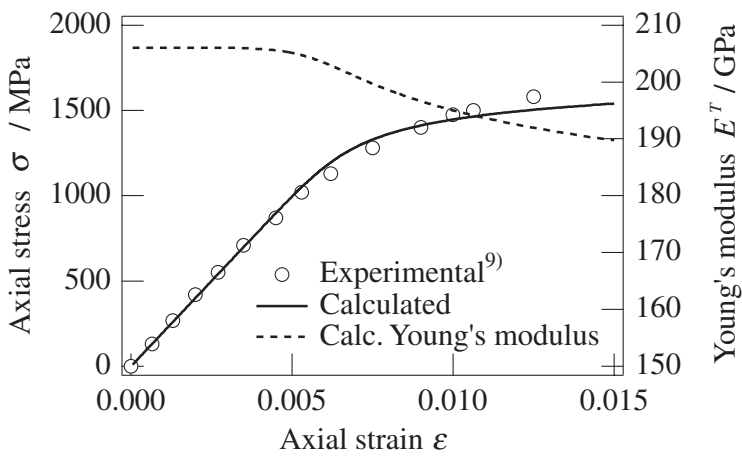

(a) Uniaxial tension

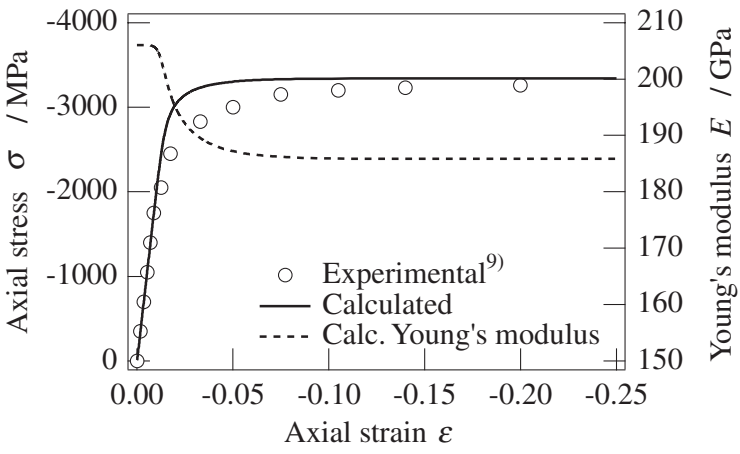

(b) Uniaxial compression

Fig. 2 Uniaxial loading of tool steel SKD11.

because of the closure effect of microcracks. As a result, the strength and toughness under tension are known to be lower than those under compression as observed in the experimental results in Figs. 2(a) and (b).

In the present paper, we employ the second rank symmetric damage tensor $\boldsymbol{D}$ for the description of the mechanical effect of the three-dimensionally distributed microcracks in the material as ${ }^{14-16)}$

$$
\boldsymbol{D}=\sum_{I=1}^{3} D_{I}\left(\boldsymbol{p}_{I} \otimes \boldsymbol{p}_{I}\right),
$$

where $D_{I}$ and $\boldsymbol{p}_{I}(I=1,2$ and 3$)$ are the principal value and principal direction of the damage variable $\boldsymbol{D}$, respectively.

\subsection{Description of unilateral characteristic of tool material}

Descriptions of the unilateral property of material damage have been proposed by some researchers. In the present paper, we introduce the modified Cauchy stress tensor as ${ }^{15,16)}$

$$
\overline{\boldsymbol{\sigma}}=\sum_{I=1}^{3}\left\langle\sigma_{I}\right\rangle\left(v_{I} \otimes v_{I}\right),
$$

where \langle\rangle is the Macauley bracket, and $\sigma_{I}$ and $v_{I}(I=1,2$ and $3)$ are the principal values and principal direction of stress tensor $\sigma$, respectively.

The modified stress tensor can be written in the global Cartesian coordinate system $x_{i}(i=1,2$ and 3$)$ as $^{16)}$

$$
\begin{aligned}
\bar{\sigma}_{i j} & =B_{i j k l} \sigma_{k l} \\
B_{i j k l} & =\sum_{K=1}^{3} h\left(\sigma_{K}\right) Q_{i K} Q_{j K} Q_{K k} Q_{K l},
\end{aligned}
$$


where $h\left(\sigma_{K}\right)$ is the Heaviside unit function for the principal stress $\sigma_{K}$, and $\boldsymbol{Q}$ is the direction cosine between the global Cartesian coordinate system $x_{i}$ and the principal stress coordinate system.

By introducing the modified stress tensor of eq. (3) combined with the damage variable $\boldsymbol{D}$ into the constitutive equations of the materials, the effect of the damage can be deactivated if the stress is compressive.

\subsection{Gibbs thermodynamic potential}

The isotropic hardening law is employed for the description of the plastic behavior of the tool material. Then, we employ a scalar isotropic hardening variable $r$.

The Gibbs thermodynamic potential of the tool materials per unit mass $\Gamma(\boldsymbol{\sigma}, \overline{\boldsymbol{\sigma}}, \boldsymbol{D}, r)$ can be postulated to consist of three parts as

$$
\Gamma(\boldsymbol{\sigma}, \overline{\boldsymbol{\sigma}}, \boldsymbol{D}, r)=\Gamma^{e}(\boldsymbol{\sigma})+\Gamma^{D}(\boldsymbol{\sigma}, \overline{\boldsymbol{\sigma}}, \boldsymbol{D})+\Gamma^{p}(r),
$$

where $\Gamma^{e}(\boldsymbol{\sigma}), \Gamma^{D}(\boldsymbol{\sigma}, \overline{\boldsymbol{\sigma}}, \boldsymbol{D})$ and $\Gamma^{p}(r)$ are the complementary energy, the complementary energy affected by the material damage, and the potential related to the plastic deformation, respectively.

The complementary energy is given by postulating the linear isotropic elastic material as follows:

$$
-\Gamma^{e}(\boldsymbol{\sigma})=\frac{1+v_{0}^{T}}{2 E_{0}^{T}} \operatorname{tr}(\boldsymbol{\sigma} \cdot \boldsymbol{\sigma})-\frac{v_{0}^{T}}{2 E_{0}^{T}}(\operatorname{tr} \boldsymbol{\sigma})^{2}
$$

where $E_{0}^{T}$ and $v_{0}^{T}$ are Young's modulus and Poisson's ratio at the initial undamaged state. In eq. (7) and hereafter, the minus sign of the components of the Gibbs thermodynamic potential are arranged in order to fit the present theory to the general thermodynamic theory.

For the elastic-damage complementary energy $\Gamma^{D}(\boldsymbol{\sigma}, \overline{\boldsymbol{\sigma}}, \boldsymbol{D})$, the following form is given by assuming the linear function of the damage variable $\boldsymbol{D}$ combined with the stress tensor or the modified stress tensor as

$$
-\Gamma^{D}(\boldsymbol{\sigma}, \overline{\boldsymbol{\sigma}}, \boldsymbol{D})=\vartheta_{1}(\operatorname{tr} \boldsymbol{\sigma})^{2} \operatorname{tr} \boldsymbol{D}+\vartheta_{2} \operatorname{tr}(\overline{\boldsymbol{\sigma}} \cdot \overline{\boldsymbol{\sigma}} \cdot \boldsymbol{D}),
$$

where $\vartheta_{1}$ and $\vartheta_{2}$ are the material constants. Let us note that the modified stress tensor $\bar{\sigma}$ is not used in the first term of the right side of eq. (8) in order to avoid the discontinuity of the constitutive equation by changing the sign of the stress under non-proportional loading conditions.

For the other terms $\Gamma^{p}(r)$ in eq. (6), we postulate the following expression in order to express the plastic deformation of the common metal materials:

$$
\Gamma^{p}(r)=R_{\infty}\left[r+\frac{1}{b} \exp (-b r)\right]
$$

where $R_{\infty}$ and $b$ are material constants.

\subsection{Elastic-damage constitutive equation and thermo- dynamic conjugate forces}

According to the conventional procedure of the thermodynamic formulation, ${ }^{11,13,14,16)}$ the elastic constitutive equation of the damaged material can be obtained as follows:

$$
\begin{aligned}
\boldsymbol{\varepsilon}^{e}= & -\frac{\partial \Gamma}{\partial \boldsymbol{\sigma}}=-\frac{\partial \Gamma^{e}}{\partial \boldsymbol{\sigma}}-\frac{\partial \Gamma^{D}}{\partial \boldsymbol{\sigma}} \\
= & \frac{1+v_{0}^{T}}{E_{0}^{T}} \boldsymbol{\sigma}-\frac{\nu_{0}^{T}}{E_{0}^{T}}(\operatorname{tr} \boldsymbol{\sigma}) \boldsymbol{I} \\
& +2 \vartheta_{1}(\operatorname{tr} \boldsymbol{D})(\operatorname{tr} \boldsymbol{\sigma})+\vartheta_{2}(\overline{\boldsymbol{\sigma}} \cdot \boldsymbol{D}+\boldsymbol{D} \cdot \overline{\boldsymbol{\sigma}}): \frac{\partial \overline{\boldsymbol{\sigma}}}{\partial \boldsymbol{\sigma}}
\end{aligned}
$$

The first and second terms of the right side of eq. (10) correspond to the linear isotropic elastic constitutive equation. The third and fourth terms express the effects of the isotopic damage and anisotropic damage on the elastic behavior of the material, respectively.

The thermodynamic conjugate forces, $\boldsymbol{Y}$, and $R$ of the internal state variables $\boldsymbol{D}$ and $r$ on the other hand, can be derived as

$$
\begin{aligned}
& \boldsymbol{Y}=-\frac{\partial \Gamma^{D}}{\partial \boldsymbol{D}}=\vartheta_{1}(\operatorname{tr} \boldsymbol{\sigma})^{2} \boldsymbol{I}+\vartheta_{2}(\overline{\boldsymbol{\sigma}} \cdot \overline{\boldsymbol{\sigma}}) \\
& R=-\frac{\partial \Gamma^{p}}{\partial r}=R_{\infty}[1-\exp (-b r)] .
\end{aligned}
$$

\subsection{Plastic-damage constitutive equation}

For the tool material considered in the present paper, the effect of the damage on plastic deformation is considered by the use of the effective stress $\tilde{\sigma}$ that describes the enlarged stress effect by the existence of the damage.

The yield surface $f$ is given as follows by use of equivalent stress that in turn is defined by the effective stress $\tilde{\boldsymbol{\sigma}}$ defined as eq. (14):

$$
\begin{aligned}
& f=\sigma_{e q}-\sigma_{Y 0}-R=0 \\
& \sigma_{e q}=\left[\frac{3}{2} \tilde{\boldsymbol{\sigma}}^{\prime}: \tilde{\boldsymbol{\sigma}}^{\prime}\right]^{1 / 2}
\end{aligned}
$$

where $\sigma_{Y 0}$ is the yield stress under the non-damaged state. The symbol (') denotes the deviatoric part. Equation (13) can be attributed to the Mises yield surface under the nondamaged state.

The effective stress $\tilde{\boldsymbol{\sigma}}$ is given as

$$
\begin{gathered}
\tilde{\boldsymbol{\sigma}}=\boldsymbol{M}(\boldsymbol{D}): \boldsymbol{\sigma} \\
{[M(D)]_{i j k l}=\frac{1}{2}\left(\delta_{i k} \delta_{j l}+\delta_{i l} \delta_{j k}\right)} \\
+c^{p}\left(D_{i k} \delta_{j l}+\delta_{i k} D_{j l}+D_{i l} \delta_{j k}+\delta_{i l} D_{j k}\right) .
\end{gathered}
$$

As observed in eq. (16), $\boldsymbol{M}(\boldsymbol{D})$ is a fourth rank symmetric tensor with damage tensor $\boldsymbol{D}$ as an argument. Moreover, $c^{p}$ is a material constant.

By assuming the associate flow rule and the normality law, the yield function of eq. (13) furnishes the rate of the plasticdamage constitutive equation as

$$
\begin{aligned}
& \dot{\boldsymbol{\varepsilon}}^{p}=\dot{\lambda} \frac{\partial f}{\partial \boldsymbol{\sigma}}=\dot{\lambda} \frac{3}{2 \sigma_{e q}} \boldsymbol{M}(\boldsymbol{D}): \tilde{\boldsymbol{\sigma}}^{\prime} \\
& \dot{\boldsymbol{r}}=-\dot{\lambda} \frac{\partial f}{\partial R}=\dot{\lambda},
\end{aligned}
$$

where the unknown multiplier $\dot{\lambda}$ can be determined from the consistency condition $\dot{f}=0$ as

$$
\dot{\lambda}=\left(\frac{\partial f}{\partial \boldsymbol{\sigma}}: \dot{\boldsymbol{\sigma}}+\frac{\partial f}{\partial \boldsymbol{D}}: \dot{\boldsymbol{D}}\right) /\left\{b_{0}\left(R_{\infty}-R\right)\right\} .
$$




\subsection{Damage evolution equation}

We assume that the damage variable $\boldsymbol{D}$ develops along the outer normal direction of the damage potential $F_{D}$ in the field of damage conjugate force $\boldsymbol{Y}$.

For the damage potential $F_{D}$, the following simple form is assumed as

$$
F_{D}(\boldsymbol{Y})=\sqrt{\boldsymbol{Y}: \boldsymbol{Y}} .
$$

Then, the evolution of damage can be prescribed as

$$
\dot{\boldsymbol{D}}=\dot{\beta} \frac{\partial F_{D}}{\partial \boldsymbol{Y}}
$$

where $\dot{\beta}$ express the magnitude of the damage development. In order to describe the fatigue damage behavior, we assume that the damage develops when the damage potential $F_{D}$ is larger than the threshold value $F_{D 0}$, and $\dot{F}_{D}$ is positive. Then, $\dot{\beta}$ can be given as

$$
\dot{\beta}=\frac{n_{D}}{K_{D}}\left\langle\frac{F_{D}-F_{D 0}}{K_{D}}\right\rangle^{n_{D}-1}\left\langle\dot{F}_{D}\right\rangle,
$$

where $n_{D}$ and $K_{D}$ are material constants.

The magnitude of the damage $\beta$ in eq. (22) is used as an equivalent damage variable in the present study. The relation between $\beta$ and $\boldsymbol{D}$ is given from eqs. (20) and (21) as

$$
\dot{\beta}=\sqrt{\dot{\boldsymbol{D}}: \dot{\boldsymbol{D}}}
$$

\subsection{Material constants of tool material}

In the present paper, the material constants of the constitutive and damage evolution equations for tool material SKD11 (JIS) or D2 (AISI) (hardness: 60HRC) are determined so that the equations can express the experimental results of tensile and compressive loading tests of SKD11 by Nakane et al. ${ }^{9)}$ as

$$
\begin{aligned}
& E_{0}^{T}=206.2 \mathrm{GPa}, \quad v_{0}^{T}=0.285, \\
& n_{D}=3.387, \quad K_{D}=1.415, F_{D 0}=0.1898, \\
& \vartheta_{1}=7.785 \times 10^{-8}, \quad \vartheta_{2}=4.502 \times 10^{-7}, \\
& c_{p}=47.42, \quad \sigma_{Y 0}=2103 \mathrm{MPa}, \\
& b=1650, \quad R_{\infty}=106800 \mathrm{MPa} .
\end{aligned}
$$

Furthermore, the critical damage $\beta_{c r}$ at which the material elements are fractured in the local approach of fracture is given as

$$
\beta_{c r}=0.5
$$

\subsection{Comparison with experimental results of tension and compression tests}

Figure 2(a) shows the experimental ${ }^{9)}$ and calculated results of stress-strain curves under uniaxial tension. The tensile strength $\sigma_{u t}$ is about $1700 \mathrm{MPa}$. In this figure, calculated result of the change in Young's modulus $E$ is also shown. We can observe the good agreement between the experimental and calculated results. We can also observe a decrease in Young's modulus. This result comes from the development of damage.

Figure 2(b) shows the experimental and calculated results of stress-strain curves under uniaxial compression. In this figure, calculated result of the change in Young's modulus $E$ is also shown. The compressive strength of the material $\sigma_{u c}$ is approximately $3200 \mathrm{MPa}$, about 1.9 times as much as $\sigma_{u t}$. We can observe that the proposed constitutive equation can describe the salient unilateral property of the tool material.

As observed in Fig. 2(b), the calculated results cannot describe the experimental result at a small strain range. This may be because of the assumption of the form of the isotropic hardening. The modification will be a future problem.

\section{Analysis of Fatigue Crack of Cold Forward Extrusion Die}

\subsection{Geometry and discretization of analyzed model}

For the application of the local approach of fracture to the cold forging tool, the analysis of the process of fatigue crack initiation and growth is performed using the elastic-plasticdamage constitutive equation proposed in chapter 3 .

A commercial FE code MARC K7 is used for the calculation. The constitutive and damage evolution equations of the die material are implemented by the user subroutines provided in the code.

Figure 4 shows the geometry and the discretization model of the cold forward extrusion. The half parts of the die and the workpiece are depicted in this figure by considering the axisymmetry of the object. The variable $l$ in Fig. 3(a) denotes the length along the die surface from the top of the die, which is used for the indication of the position of the die surface.

As the finite elements, we use the axisymmetric triangular and axisymmetric quadrilateral elements for the extrusion die and the workpiece, respectively. The numbers of elements are 3092 and 2739 for the die and the workpiece, respectively. The numbers of nodes are 1606 and 4442 for the die and the workpiece, respectively.

In practical cold forward extrusion, the stress ring restrains the expansion of the die. In the present calculations, instead of the modeling of the stress ring, the displacement of the periphery of the die is restrained in order to save the calculation cost.

As the contact condition, we use the Coulomb friction with the friction coefficient $\mu=0.1$ at the interface between the die and the workpiece.

\subsection{Elastic-plastic constitutive equation of workpiece material}

SS400 in JIS is employed as a workpiece material in the present study.

As the elastic property, we assume the linear isotropic elastic material. Young's modulus $E_{0}^{W}$ and Poisson's ratio $v_{0}^{W}$ are given as

$$
E_{0}^{W}=206.1 \mathrm{GPa}, \quad \nu_{0}^{W}=0.289 .
$$

For the plastic property of the material, we assume the exponential work hardening of the material and give the relation between the true stress $\sigma$ and true strain $\varepsilon$ as

$$
\sigma=285.0+461.0\left(\varepsilon-1.38 \times 10^{-4}\right)^{0.286} .
$$




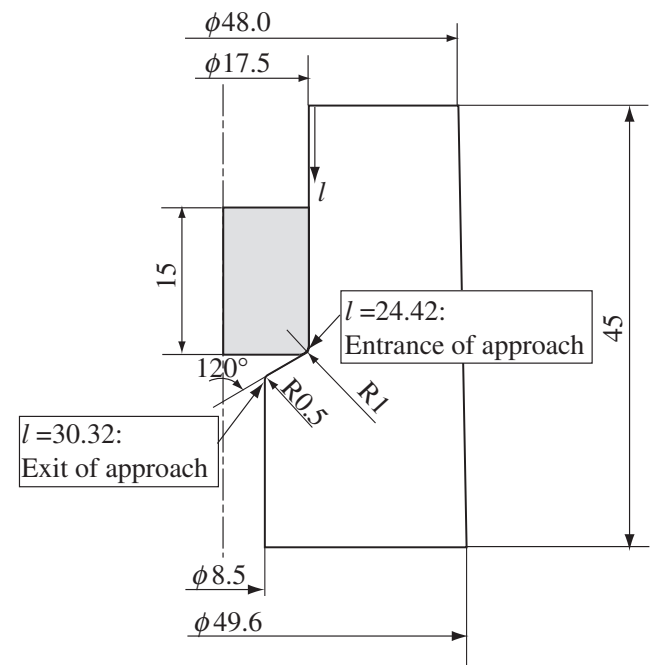

(a) Geometry of model

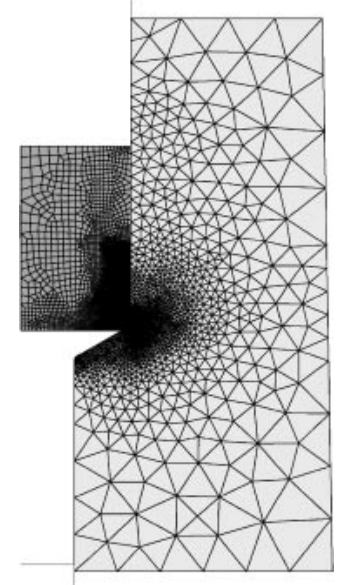

(b) Discretized model

Fig. 3 Analyzed model of cold forward extrusion.

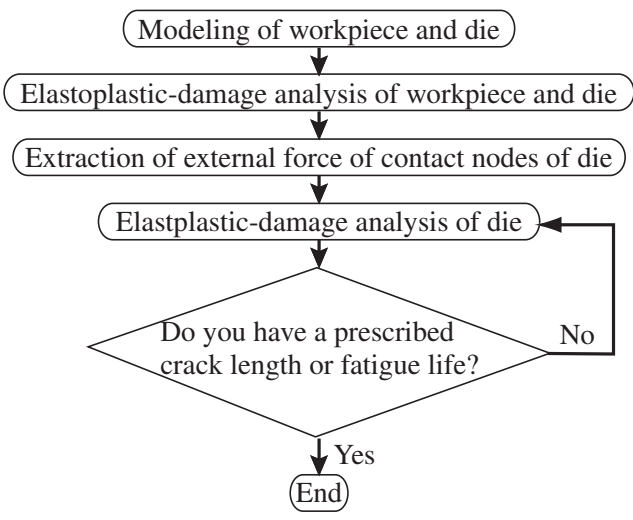

Fig. 4 Flowchart for fatigue life estimation.

\subsection{Procedure of analysis for cyclic extrusion}

Generally, the calculation considering the large deformation as well as the contact between the die and the workpiece with friction is complicated, and it may not be appropriate to calculate the cyclic operation process from the viewpoint of calculation cost.
Therefore, in the present study, we perform the fatigue crack analysis according to the following procedure as shown in Fig. 4.

i) The elastic-plastic analysis of a cold forward extrusion process is first performed. The punch travel $s$ is up to $3 \mathrm{~mm}$. After the extrusion, the processes of the ejection of the workpiece are simulated.

ii) The extraction of the external force of the contact surface between the die and workpiece as a function of the punch displacement is conducted.

iii) The elastic-plastic-damage analysis of cyclic loading of only the die is performed by use of the external force extracted in ii) as boundary conditions of the die.

\subsection{Calculated results and discussion of analysis of single extrusion process of die-workpiece}

Figure 5 shows the normal and tangential stress on the die surface during the extrusion (punch travel $s=1,2$ and $3 \mathrm{~mm}$ ) and the knockout processes. The direction and the length of the arrows denote the direction and the magnitude of the surface stress. As observed in these figures, the normal and the tangential stress on the die surface vary as the extrusion progresses. We can also observe that the extrusion die is subjected to the normal and the tangential stress even in the knockout process.

The value of the maximal principal stress of the die considerably influences the development of the damage variable. Figure 6 shows the distribution of the maximal principal stress on the die surface at the punch travel $s=1,2$ and $3 \mathrm{~mm}$. In this figure, the variable $l$ in Fig. 3(a) is used in the abscissa. The major principal stress has peaks at $l \approx 24.5$ and $30 \mathrm{~mm}$. These positions correspond to the die radius and the outlet of the die. As the maximal values are observed at $l \approx 24.5 \mathrm{~mm}$, the fatigue crack may start in the vicinity of this position. Furthermore, since the maximal principal stress at $s=2 \mathrm{~mm}$ is larger than that at $s=3 \mathrm{~mm}$, the damage at a single extrusion process can be considered to cease to develop by $s=3 \mathrm{~mm}$.

In some studies performed so far, ${ }^{7,8)}$ the assumption of constant internal pressure in die is employed. However, the actual stress on the die surface may vary in the position and during the process. Therefore, when considering the plastic and damage behavior of the die material, the actual loading histories are necessary for more precise estimation.

\subsection{Results and discussion of the calculation of cyclic extrusion process}

For the calculation of the cyclic forward extrusion process by only the die, we divide the single extrusion process performed in section 4.4 into 90 increments. Then, the equivalent external nodal forces at a certain punch travel $s$ obtained from the calculation in section 4.4 are given at the corresponding nodes on the die surface as the boundary conditions of the die.

The elements that attain to the critical value of the equivalent damage variable $\beta_{c r}$ are regarded as fractured, and the stiffness of the elements is reduced to zero.

In the present calculation, the element first attains to the critical value of $\beta_{c r}$ at the number of extrusion $N=86$.

Figure 7 shows the distribution of the major principal 


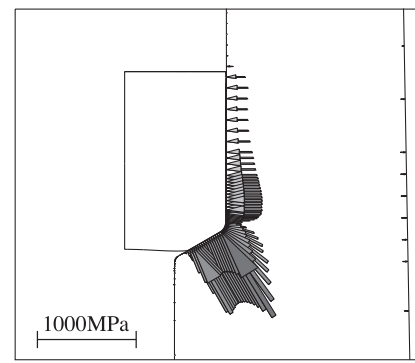

Punch travel $s=1 \mathrm{~mm}$

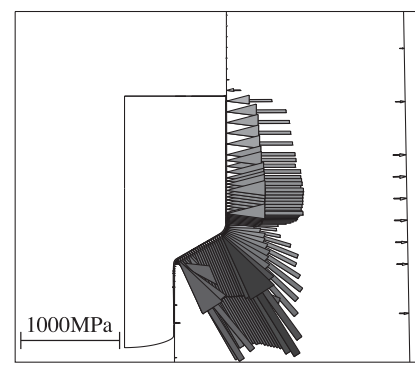

Punch travel $s=2 \mathrm{~mm}$

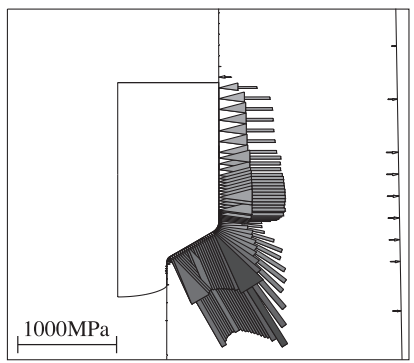

Punch travel $s=2 \mathrm{~mm}$

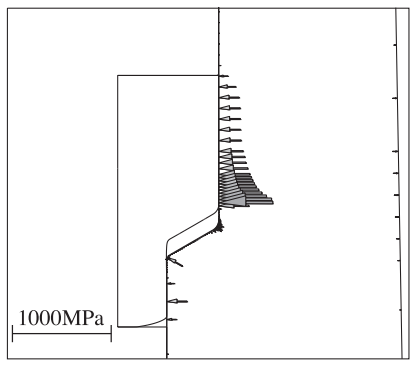

Ejection

(a) Normal stress

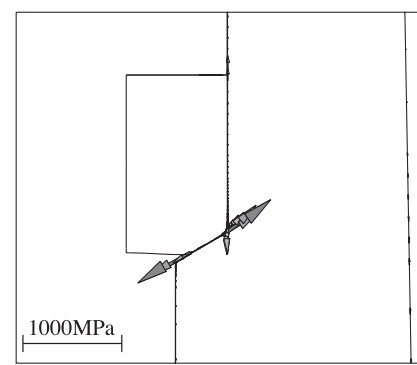

Punch travel $s=1 \mathrm{~mm}$

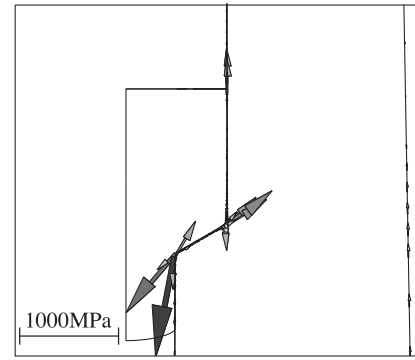

Punch travel $s=2 \mathrm{~mm}$

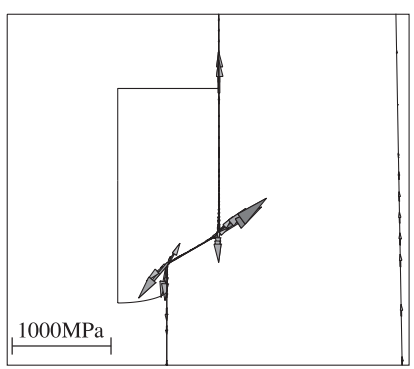

Punch travel $s=2 \mathrm{~mm}$

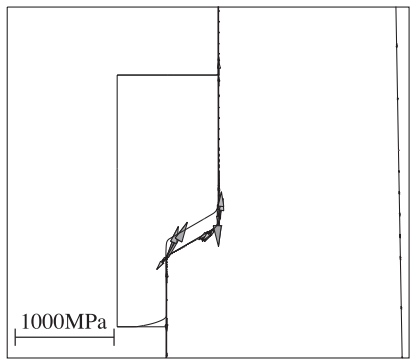

Knock out (b) Shear stress

Fig. 5 Extracted contact normal and shear stresses shown by arrows.

stress $\sigma_{m a j}$ at the punch travel $s=3 \mathrm{~mm}$ and the extrusion number $N=85$, just before the number of the first onset of the fatigue crack.

The black and gray color of the arrows denotes the positive (tensile) and the negative (compressive) values of $\sigma_{m a j}$, respectively. As observed in this figure, the positive value of the major principal stress is not observed except in the region of the die radius. From this result, the development of damage can be considered to be dominant in this region.

This positive major principal stress is caused by the bending moment by the compressive and shear stresses on the die surface. The extent of the positive major principal stress

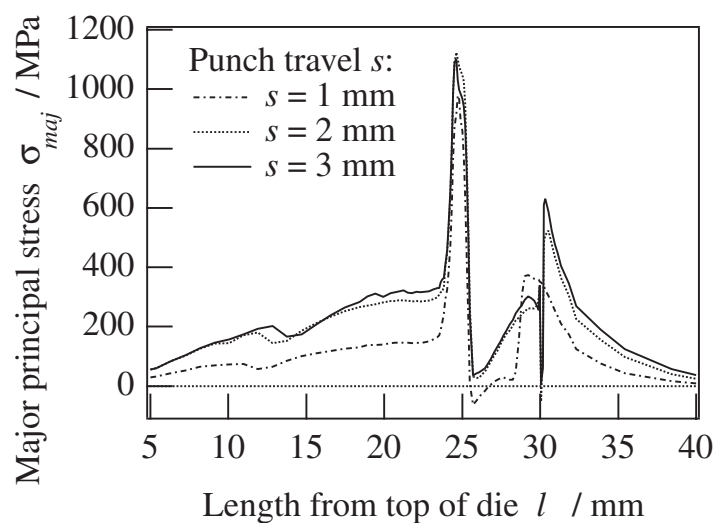

Fig. 6 Distribution of major principal stress along die surface.

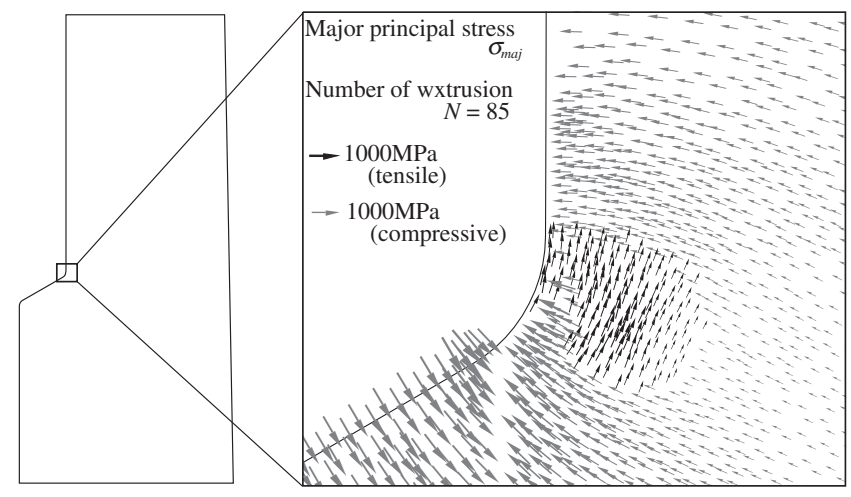

Fig. 7 Direction and magnitude of major principal stress at number of extrusion $N=85$.

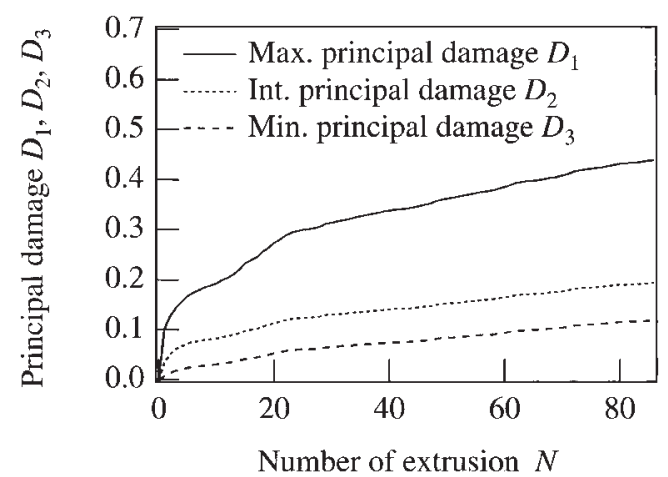

Fig. 8 Change in principal damage values at initially fractured element to number of extrusion $N$.

is highly affected by the die angle and the die radius.

On the other hand, it is also observed that the negative major principal stress is dominant in most regions of the extrusion die. The damage development in the region is much less than in the region where the positive major principal stress is dominant.

It is necessary for the precise estimation of the fatigue crack onset and growth to employ the elastic-plastic constitutive equations taking the unilateral property into account.

Figure 8 shows the change of the three principal values of the damage variable $\boldsymbol{D}$ of the finite element which is the first to fracture at the number of extrusion $N=86$. As observed in 


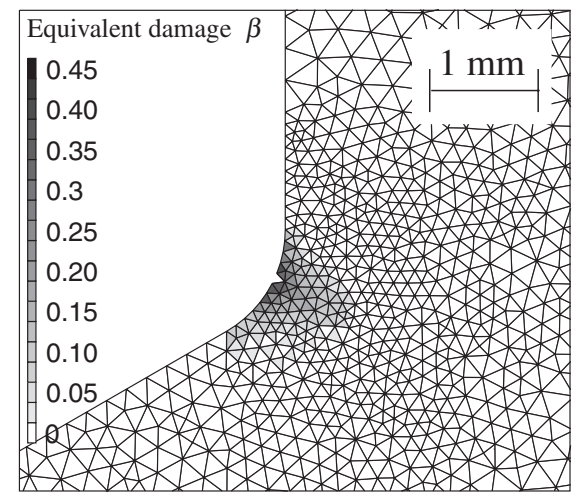

(a) $N=100$

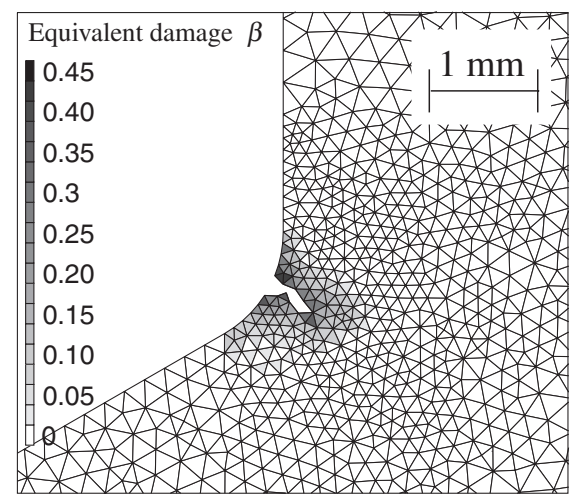

(b) $N=200$

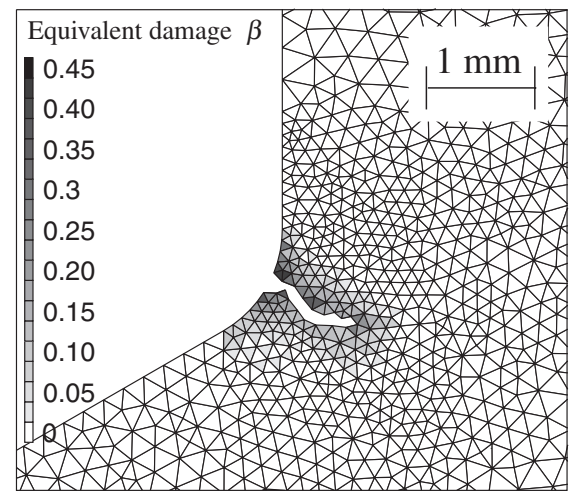

(c) $N=300$

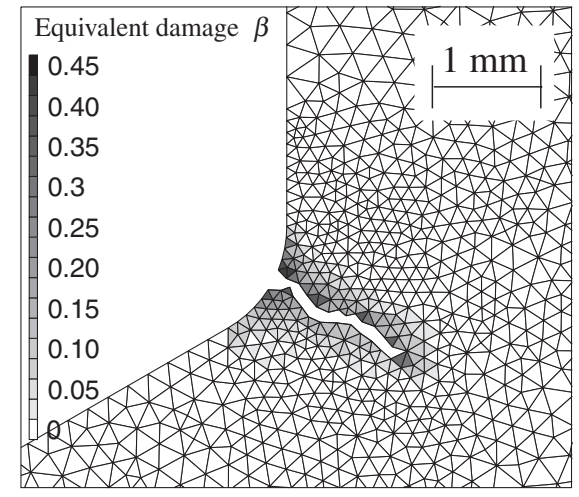

(d) $N=500$

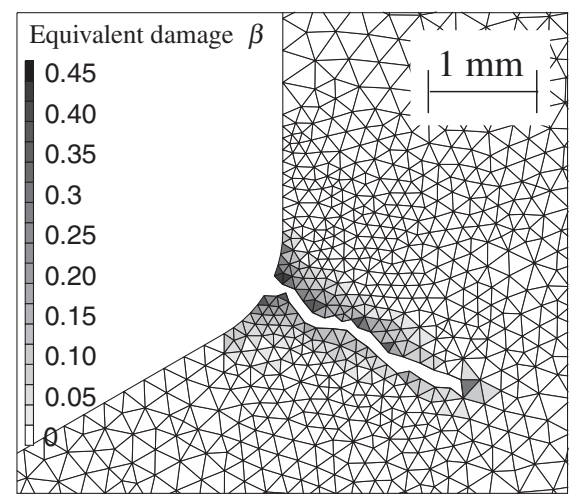

(e) $N=1000$

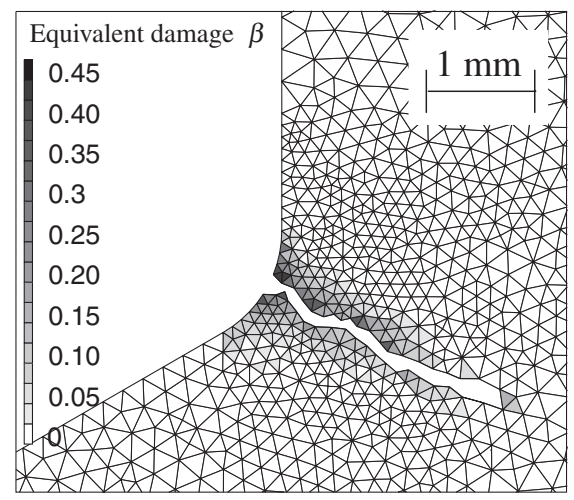

(f) $N=2500$

Fig. 9 Aspect of crack propagation and distribution of equivalent damage $\beta$.

this figure, the development of the damage is anisotropic as $D_{1}$ is dominant. The principal direction of $D_{1}$ almost corresponds to the direction of the positive major principal stress.

Figure 9 shows the aspect of the crack propagation and the distribution of the equivalent damage variable $\beta$ at each $N$ until $N=2500$. From this figure, we can observe that the crack initiates at near the center of the die radius, and propagates along the direction perpendicular to the surface of the die radius. This direction nearly corresponds to the principal direction of the positive major principal stress.

Furthermore, the equivalent damage variable can be observed to develop mainly at the element located at the crack tip. Since stiffness of the fractured elements is reduced to zero, the stress concentration at the crack tip can be expressed properly.
Figure 10 shows the comparison of the change in the calculated crack length $a$ with $N$ with the experimental results by Reiss. ${ }^{1)}$ We can observe that $a$ shows the good agreement with the calculated results till $N$ becomes approximately 2000. Afterward, however, the calculated rate of the crack propagation decreases, and the crack length $a$ becomes smaller than that of the experiments. The calculated crack length stops propagating at $a \approx 3 \mathrm{~mm}$ that corresponds to the length of the region where the positive major principal stress is observed as shown in Fig. 7. Such a phenomenon of non-propagating of the crack is also pointed out by Reiss ${ }^{1)}$ and Sonsöz and Tekkaya. ${ }^{7)}$

Further crack propagation till the final failure of the die in the experiment has been observed. It is necessary to consider the effect of the penetration of the workpiece or lubricants into the crack to cause the further propagation of the fatigue 


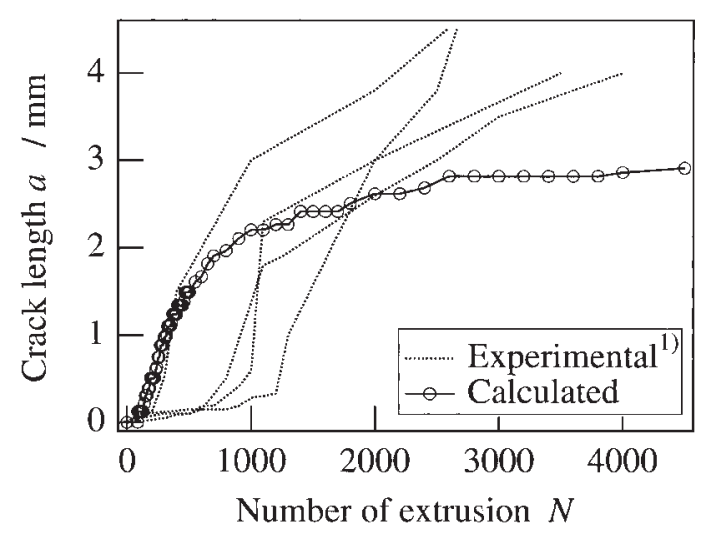

Fig. 10 Result of crack length $a$ as a function of number of extrusion $N$.

crack. There have been no reports on a calculation for the crack propagation that takes the penetration phenomenon into account, including the present study.

\section{Concluding Remarks}

The local approach of fracture based on CDM with FEM has been shown to be a promising method for the unified treatment of the initiation and growth of a crack in structures. In the present study, we apply this approach to the calculation of the fatigue crack initiation and growth of the cold forward extrusion die in order to verify this approach to the precise estimation of the service life of the cold forging tool.

Concluding remarks obtained from the present study can be summarized as follows:

(1) By describing the material damage that has the unilateral nature by use of the damage variable of the second rank symmetric tensor, the elastic-plastic-damage constitutive equation of the cold forging tool was formulated. The proposed equation can describe the experimental results of the large difference in strength under uniaxial tension and compression of cold tool material SKD11.

(2) FE analysis of the die and workpiece on the extrusionejection processes of cold forward extrusion was performed.
As a result, it was clarified that the distributions of the normal and shear stresses are complicated, and they change as the punch travels.

(3) By performing the analysis of the fatigue crack initiation and propagation of the extrusion die under the cyclic extrusion process by use of the history of the loading of the die surface, the crack first initiates at the region where the maximal tensile principal stress is observed, and propagates in the direction perpendicular to that of the tensile principal stress.

(4) The calculated result on the change in the crack length $a$ to the number of extrusion $N$ shows good agreement with the experimental result until the $N$ is about 2000. Afterwards, the growth of $a$ is stagnated because the effect of the penetration of the workpiece or lubricants into the crack is not taken into account on the present calculation.

\section{REFERENCES}

1) W. Reiss: CIRP Ann. 36 (1987) 155-160.

2) U. Engel and M. Hänsel: Proc. 3rd ICTP 1 (1990) 355-360.

3) M. Hänsel, U. Engel and M. Geiger: Mixed-Mode Fatigue and Fracture, (Mechanical Engineering Publication, 1993) pp. 3-21.

4) L. Cser, M. Geiger, K. Lange, J. A. G. Kals and M. Hänsel: Proc. Instn. Mech. Engrs. 207 (1993) 223-239.

5) M. Knoerr, K. Lange and T. Altan: J. Mater. Process. Technol. 46 (1994) 57-71.

6) M. Geiger: CIRP Ann. 40 (1991) 303-306.

7) A. Sonsöz and A. E. Tekkaya: Int. J. Mech. Sci. 38 (1996) 527-538.

8) S. H. Ahn, T. H. Kim, B. M. Kim and J. C. Choi: J. Mater. Process. Technol. 71 (1997) 343-349.

9) T. Nakane, H. Kudo and S. Matsubara: Proc. 17th Jpn. Joint Conf. for Technol. Plasticity, (1966) 281-284 (in Japanese).

10) J. Lemaitre: Eng. Fract. Mech. 25 (1986) 523-537.

11) J. Lemaitre: A Course on Damage Mechanics (2nd Ed.), (SpringerVerlag, Berlin, 1996).

12) S. Murakami and Y. Liu: Mat. Sci. Res. Int. 2 (1996) 131-142.

13) J. Lemaitre and J.-L. Chaboche: Mechanics of Solid Materials, (Cambridge University Press, 1985).

14) S. Murakami: JSME Int. J. 30 (1987) 701-710.

15) S. Murakami: J. Appl. Mech., Trans. ASME 55 (1988) 280-286.

16) K. Hayakawa and S. Murakami: Int. J. Damage Mech. 6 (1997) 333363. 